\begin{tabular}{|l|}
\hline A\&A manuscript no. \\
(will be inserted by hand later) \\
\hline Your thesaurus codes are: \\
$\mathbf{0 6}(\mathbf{0 8 . 0 2 . 5 )}$
\end{tabular}

ASTRONOMY AND ASTROPHYSICS 27.10 .2018

\title{
Symbiotic stars on Asiago archive plates ${ }^{\boxplus}$
}

\author{
Ulisse Munari ${ }^{1}$, Rajka Jurdana-Šepić ${ }^{2}$, and Dina Moro ${ }^{1}$ \\ ${ }^{1}$ Osservatorio Astronomico di Padova, Sede di Asiago, I-36012 Asiago (VI), Italy \\ 2 Physics Department, University of Rijeka, Omladinska 14, HR-51000 Rijeka, Croatia
}

Received date. ; accepted date.

\begin{abstract}
The rich plate archive of the Asiago observatory has been searched for plates containing the symbiotic stars AS 323, Ap 3-1, CM Aql, V1413 Aql (= AS 338), V443 Her, V627 Cas (= AS 501) and V919 Sgr. The program objects have been found on 602 plates, where their brightness has been estimated against the $U B V(R I)_{\mathrm{C}}$ photometric sequences calibrated by Henden and Munari (2000).

AS 323 is probably eclipsing, with a preliminary $\mathrm{P}=197.6$ day period. If confirmed, it would be the shortest orbital period known among symbiotic stars. CM Aql does not seem to undergo a series of outbursts, its lightcurve being instead modulated by a large amplitude sinusoidal variation with a $\mathrm{P} \sim 1058$ day period. V627 Cas presents a secular trend in agreement with the possible post-AGB nature of its cool component.
\end{abstract}

Key words: Binaries: symbiotic

\section{Introduction}

The time scale of variability for symbiotic stars is quite long: the orbital periods range from $\sim 1$ year up to several decades while rise and decay from an outburst may take anything from a few years to more than a century (cf. Kenyon 1986).

Such long time scales tend to discourage stand-alone photometric campaigns from a single Observatory, which could pay dividends only after ten or more years. Most of the current photometric investigations of symbiotic stars therefore try to assemble as much as possible data from the widest set of current and archival sources. Template examples are the reconstruction of the 1890-1996 lightcurve of YY Her by Munari et al. (1997) and the 1885-1988

Send offprint requests to: U.Munari

* Table 1 is only available in electronic form at the CDS via anonymous ftp to cdsarc.u-strasbg.fr (130.79.128.5) or via http://cdsweb.u-strasbg.fr/cgi-bin/qcat?J/A+A/ lightcurve of CH Cyg by Mikolajewski et al. (1990). Both required a huge effort in locating and measuring historical material in plate archives around the world.

Henden and Munari $(2000,2001)$ have so far provided accurate and extended $U B V(R I)_{\mathrm{C}}$ photometric comparison sequences around 40 symbiotic stars, intended to assist both present time photometry as well as measurement of photographic plates from historical archives. They should stimulate small observatories and/or occasional observers to obtain new data as well as to encourage those with access to old plate archives to search for valuable historical data. Assembling such data (obtained at various Observatories against the same comparison sequences to minimize systematic errors) will result in a much better understanding of the photometric evolution and therefore the physical nature of this intriguing class of interacting binaries.

In this paper we present the results of digging the Asiago plate archive for seven symbiotic stars: AS 323, Ap 31, CM Aql, V1413 Aql (= AS 338), V443 Her, V627 Cas $(=$ AS 501) and V919 Sgr.

\section{Data acquisition}

Two Schmidt telescopes were operated at Asiago observatory. The smaller one $(40 / 50 \mathrm{~cm}, 100 \mathrm{~cm}$ focal length) collected 20417 plates from 1958 to 1992, and the larger one (67/92 cm, $208 \mathrm{~cm}$ focal length) 18811 plates from 1965 to 1998 . The Asiago Schmidt plate collection thus span 40 years. The majority of the plates match the $B$ band, but the $U, V, R_{C}$ and $I_{C}$ bands are well represented too.

The plates are typically filed in the archive logs with the coordinates of the object to which they were aimed, that generally does not lay in the plate center (which is instead usually the case for the guiding star).

Therefore, for a given program star, we initially selected from the archive logs the plates to inspect as if they were covering a $2 \times 2$ wider area. A subsequent visual inspection of all the selected plates separated those actually containing the program star (602 plates) from the others.

We then proceeded to estimate at an high quality binocular microscope the magnitude of the program star 
Table 1. The $U B V(R I)_{\mathrm{C}}$ magnitudes of the program stars estimated on the Asiago archive plates. The date is given in the year/month/day format, the heliocentric JD is $H J D=J D_{\odot}-2400000$ and the magnitude is estimated in steps of 0.05 mag.

\begin{tabular}{|c|c|c|c|c|c|c|c|c|c|c|c|c|c|c|}
\hline \multicolumn{3}{|c|}{ date } & $H J D$ & mag & & late & & HJD & mag & & late & & HJD & mag \\
\hline \multicolumn{5}{|c|}{ AS 323} & \multicolumn{5}{|c|}{ AS 323} & \multicolumn{5}{|c|}{ Ap 3-1 } \\
\hline 1961 & 08 & 05 & 37517.414 & $\mathrm{~B}=15.45$ & 1973 & 09 & 19 & 41945.339 & $\mathrm{~B}=15.50$ & 1971 & 11 & 06 & 41262.238 & $B=18.20$ \\
\hline 1961 & 08 & 06 & 37518.415 & $B=15.45$ & 1973 & 09 & 27 & 41953.275 & $\mathrm{R}=12.50$ & 1971 & 11 & 20 & 41276.210 & $\mathrm{I}=13.40$ \\
\hline 1961 & 08 & 07 & 37519.424 & $\mathrm{~B}=15.50$ & 1973 & 09 & 27 & 41953.293 & $\mathrm{~V}=14.20$ & 1971 & 11 & 20 & 41276.230 & $B=18.20$ \\
\hline 1961 & 08 & 08 & 37520.420 & $\mathrm{~B}=15.50$ & 1973 & 09 & 27 & 41953.309 & $\mathrm{~B}=15.30$ & 1972 & 08 & 03 & 41533.402 & $\mathrm{I}=13.20$ \\
\hline 1961 & 08 & 09 & 37521.442 & $B=16.60$ & 1973 & 09 & 28 & 41954.272 & $\mathrm{R}=12.50$ & 1972 & 08 & 13 & 41543.477 & $\mathrm{I}=13.10$ \\
\hline 1961 & 08 & 10 & 37522.444 & $B=15.70$ & 1973 & 09 & 28 & 41954.288 & $\mathrm{R}=12.55$ & 1972 & 08 & 17 & 41547.427 & $B=18.70$ \\
\hline 1961 & 08 & 11 & 37523.467 & $\mathrm{~B}=15.35$ & 1973 & 10 & 02 & 41958.269 & $\mathrm{~V}=14.40$ & 1972 & 08 & 17 & 41547.446 & $\mathrm{I}=13.00$ \\
\hline 1961 & 08 & 12 & 37524.471 & $\mathrm{~B}=15.30$ & 1975 & 06 & 19 & 42582.517 & $\mathrm{~B}=15.50$ & 1972 & 09 & 28 & 41589.274 & $B=18.20$ \\
\hline 1961 & 08 & 17 & 37529.427 & $\mathrm{~B}=15.50$ & 1975 & 06 & 19 & 42582.527 & $\mathrm{~V}=14.45$ & 1972 & 09 & 28 & 41589.290 & $\mathrm{I}=12.45$ \\
\hline 1961 & 08 & 18 & 37530.448 & $B=15.55$ & 1975 & 07 & 02 & 42596.454 & $B=15.45$ & 1972 & 10 & 04 & 41595.323 & $\mathrm{I}=13.00$ \\
\hline 1961 & 08 & 29 & 37541.354 & $B=15.70$ & 1975 & 07 & 02 & 42596.466 & $V=15.45$ & 1972 & 10 & 04 & 41595.344 & $B=18.60$ \\
\hline 1961 & 08 & 30 & 37542.371 & $\mathrm{~B}=15.80$ & 1975 & 08 & 03 & 42628.441 & $V=14.40$ & 1972 & 10 & 31 & 41622.270 & $\mathrm{I}=13.10$ \\
\hline 1961 & 08 & 31 & 37543.385 & $\mathrm{~B}=15.60$ & 1975 & 08 & 03 & 42628.460 & $\mathrm{~B}=15.40$ & 1972 & 10 & 31 & 41622.287 & $B=18.20$ \\
\hline 1961 & 09 & 01 & 37544.382 & $\mathrm{~B}=15.70$ & 1975 & 10 & 06 & 42692.333 & $\mathrm{~V}=14.25$ & 1972 & 11 & 02 & 41624.246 & $\mathrm{I}=13.15$ \\
\hline 1961 & 09 & 03 & 37546.338 & $B=15.70$ & 1975 & 10 & 29 & 42715.265 & $B=15.30$ & 1972 & 11 & 02 & 41624.263 & $B=18.50$ \\
\hline 1961 & 09 & 04 & 37547.396 & $B=15.70$ & 1975 & 10 & 29 & 42715.288 & $V=14.40$ & 1972 & 11 & 06 & 41628.246 & $\mathrm{I}=13.10$ \\
\hline 1961 & 09 & 06 & 37549.388 & $B=15.65$ & 1976 & 07 & 31 & 42991.436 & $V=14.30$ & 1972 & 11 & 06 & 41628.265 & $B=18.30$ \\
\hline 1961 & 09 & 07 & 37550.345 & $\mathrm{~B}=15.70$ & 1976 & 07 & 31 & 42991.463 & $\mathrm{~B}=15.20$ & 1973 & 08 & 18 & 41913.341 & $B=18.20$ \\
\hline 1961 & 09 & 09 & 37552.374 & $\mathrm{~B}=15.70$ & 1976 & 08 & 19 & 43010.385 & $\mathrm{~B}=15.30$ & 1973 & 08 & 18 & 41913.361 & $\mathrm{I}=13.40$ \\
\hline 1961 & 09 & 10 & 37553.378 & $B=15.70$ & 1976 & 08 & 19 & 43010.386 & $V=14.45$ & 1973 & 08 & 24 & 41919.449 & $V=17.00$ \\
\hline 1961 & 09 & 13 & 37556.341 & $B=15.75$ & 1977 & 07 & 23 & 43348.399 & $\mathrm{I}=11.95$ & 1973 & 08 & 29 & 41924.402 & $V=16.90$ \\
\hline 1961 & 09 & 15 & 37558.369 & $\mathrm{~B}=15.70$ & 1977 & 07 & 24 & 43349.469 & $\mathrm{I}=12.15$ & 1973 & 10 & 03 & 41959.371 & $\mathrm{I}=13.60$ \\
\hline 1961 & 09 & 16 & 37559.358 & $\mathrm{~B}=15.75$ & 1977 & 07 & 24 & 43349.486 & $V=14.40$ & 1973 & 10 & 17 & 41973.345 & $\mathrm{I}=13.50$ \\
\hline 1961 & 09 & 17 & 37560.403 & $\mathrm{~B}=15.40$ & 1977 & 08 & 10 & 43366.371 & $\mathrm{~V}=14.35$ & 1974 & 07 & 22 & 42251.475 & $B=18.20$ \\
\hline 1961 & 09 & 26 & 37569.292 & $B=15.10$ & 1977 & 08 & 10 & 43366.387 & $\mathrm{I}=12.15$ & 1974 & 07 & 29 & 42258.482 & $\mathrm{I}=13.00$ \\
\hline 1961 & 10 & 02 & 37575.341 & $B=15.40$ & 1977 & 09 & 06 & 43393.317 & $\mathrm{I}=12.10$ & 1974 & 08 & 14 & 42274.371 & $B=18.50$ \\
\hline 1961 & 10 & 05 & 37578.327 & $B=15.35$ & 1977 & 10 & 04 & 43421.265 & $B=15.30$ & 1974 & 08 & 14 & 42274.393 & $\mathrm{I}=13.35$ \\
\hline 1961 & 10 & 09 & 37582.313 & $B=15.40$ & 1977 & 10 & 04 & 43421.286 & $\mathrm{I}=12.10$ & 1974 & 09 & 09 & 42300.425 & $B=18.20$ \\
\hline 1961 & 10 & 10 & 37583.326 & $\mathrm{~B}=15.25$ & 1978 & 09 & 28 & 43780.389 & $\mathrm{~V}=14.35$ & 1974 & 09 & 09 & 42300.445 & $\mathrm{I}=13.65$ \\
\hline 1962 & 06 & 08 & 37824.487 & $B=15.10$ & 1978 & 09 & 28 & 43780.406 & $\mathrm{I}=12.10$ & 1974 & 09 & 13 & 42304.384 & $B=18.40$ \\
\hline 1962 & 06 & 24 & 37840.422 & $B=15.20$ & 1980 & 08 & 09 & 44461.413 & $B=16.00$ & 1974 & 09 & 13 & 42304.404 & $\mathrm{I}=13.65$ \\
\hline 1962 & 08 & 06 & 37883.430 & $B=15.35$ & 1989 & 09 & 22 & 47792.306 & $B=16.10$ & 1975 & 05 & 18 & 42550.569 & $V=17.00$ \\
\hline 1963 & 07 & 19 & 38230.463 & $\mathrm{~B}=15.35$ & 1989 & 10 & 05 & 47805.306 & $\mathrm{~B}=16.50$ & 1975 & 05 & 18 & 42550.588 & $B=18.50$ \\
\hline 1964 & 09 & 07 & 38646.403 & $\mathrm{~B}=15.35$ & 1989 & 10 & 07 & 47807.358 & $\mathrm{~B}=15.70$ & 1975 & 06 & 18 & 42582.502 & $B=18.20$ \\
\hline 1965 & 06 & 27 & 38939.453 & $B=15.35$ & 1990 & 05 & 19 & 48030.521 & $B=15.60$ & 1975 & 10 & 30 & 42716.337 & $B=18.40$ \\
\hline 1965 & 06 & 27 & 38939.468 & $B=15.35$ & 1990 & 07 & 19 & 48092.436 & $B=15.35$ & 1976 & 09 & 17 & 43039.304 & $B=18.20$ \\
\hline 1965 & 10 & 24 & 39058.258 & $\mathrm{~B}=15.35$ & 1990 & 07 & 19 & 48092.464 & $\mathrm{~V}=14.40$ & 1976 & 09 & 17 & 43039.320 & $\mathrm{I}=13.00$ \\
\hline 1966 & 05 & 26 & 39271.566 & $\mathrm{~B}=15.35$ & 1990 & 07 & 20 & 48093.448 & $\mathrm{~V}=14.45$ & 1976 & 10 & 21 & 43073.315 & $\mathrm{I}=13.00$ \\
\hline 1970 & 08 & 03 & 40802.394 & $\mathrm{~B}=15.30$ & 1990 & 08 & 21 & 48125.330 & $\mathrm{~V}=14.35$ & 1976 & 10 & 21 & 43073.336 & $B=17.50$ \\
\hline 1970 & 08 & 03 & 40802.401 & $V=14.50$ & 1990 & 08 & 23 & 48127.333 & $B=15.45$ & 1977 & 06 & 19 & 43314.471 & $B=18.20$ \\
\hline 1970 & 08 & 05 & 40804.371 & $B=15.35$ & 1991 & 07 & 15 & 48453.467 & $B=14.50$ & 1977 & 06 & 19 & 43314.493 & $\mathrm{I}=12.45$ \\
\hline 1970 & 08 & 10 & 40809.431 & $B=15.70$ & & & & & & 1977 & 07 & 18 & 43343.436 & $B=18.20$ \\
\hline 1970 & 08 & 10 & 40809.445 & $\mathrm{~V}=14.40$ & & & & Ap 3-1 & & 1977 & 07 & 19 & 43344.414 & $\mathrm{I}=12.95$ \\
\hline 1970 & 08 & 11 & 40810.427 & $\mathrm{~B}=15.30$ & & & & & & 1977 & 08 & 12 & 43368.494 & $\mathrm{I}=12.90$ \\
\hline 1970 & 08 & 11 & 40810.441 & $V=14.50$ & 1969 & 07 & 16 & 40419.449 & $B=17.70$ & 1977 & 08 & 13 & 43368.514 & $B=18.20$ \\
\hline 1970 & 09 & 06 & 40836.358 & $V=14.35$ & 1970 & 06 & 13 & 40750.511 & $\mathrm{~B}=18.40$ & 1977 & 09 & 05 & 43392.378 & $\mathrm{I}=12.85$ \\
\hline 1970 & 09 & 06 & 40836.370 & $B=15.30$ & 1970 & 07 & 05 & 40772.542 & $B=18.00$ & 1977 & 09 & 05 & 43392.399 & $B=18.20$ \\
\hline 1970 & 09 & 06 & 40836.395 & $\mathrm{~V}=14.40$ & 1970 & 07 & 28 & 40796.400 & $\mathrm{I}=13.10$ & 1977 & 09 & 09 & 43396.364 & $\mathrm{I}=13.50$ \\
\hline 1970 & 09 & 06 & 40836.409 & $\mathrm{~B}=15.30$ & 1970 & 08 & 04 & 40803.404 & $\mathrm{~B}=18.40$ & 1977 & 09 & 09 & 43396.386 & $B=18.20$ \\
\hline 1970 & 10 & 21 & 40881.267 & $B=15.40$ & 1970 & 08 & 04 & 40803.418 & $B=18.50$ & 1977 & 10 & 03 & 43420.316 & $\mathrm{I}=13.00$ \\
\hline 1970 & 10 & 21 & 40881.286 & $\mathrm{~V}=14.35$ & 1970 & 08 & 05 & 40804.493 & $\mathrm{~B}=18.20$ & 1977 & 10 & 13 & 43430.338 & $\mathrm{I}=13.00$ \\
\hline 1970 & 10 & 22 & 40882.237 & $V=14.35$ & 1970 & 08 & 24 & 40823.394 & $B=18.50$ & 1978 & 08 & 10 & 43731.468 & $V=16.90$ \\
\hline 1970 & 10 & 22 & 40882.249 & $\mathrm{~B}=15.30$ & 1970 & 09 & 06 & 40836.391 & $\mathrm{~B}=17.80$ & 1978 & 08 & 10 & 43731.494 & $\mathrm{I}=13.00$ \\
\hline 1970 & 10 & 26 & 40886.260 & $\mathrm{~V}=14.40$ & 1970 & 09 & 28 & 40858.329 & $\mathrm{~B}=18.30$ & 1978 & 08 & 30 & 43751.440 & $V=16.30$ \\
\hline 1970 & 10 & 26 & 40886.274 & $B=15.35$ & 1970 & 10 & 25 & 40885.267 & $B=18.00$ & 1978 & 08 & 30 & 43751.479 & $\mathrm{I}=12.50$ \\
\hline 1971 & 06 & 15 & 41118.484 & $\mathrm{~V}=15.35$ & 1971 & 07 & 01 & 41133.544 & $\mathrm{~B}=18.20$ & 1978 & 10 & 05 & 43787.340 & $V=16.40$ \\
\hline 1971 & 06 & 19 & 41122.497 & $B=15.30$ & 1971 & 07 & 02 & 41134.514 & $\mathrm{~B}=18.00$ & 1978 & 10 & 05 & 43787.362 & $\mathrm{I}=13.15$ \\
\hline 1971 & 10 & 18 & 41243.262 & $V=14.30$ & 1971 & 07 & 02 & 41134.527 & $\mathrm{~B}=18.00$ & 1978 & 10 & 29 & 43811.324 & $V=17.00$ \\
\hline 1971 & 10 & 20 & 41245.236 & $\mathrm{I}=12.05$ & 1971 & 09 & 11 & 41206.337 & $\mathrm{~B}=18.20$ & 1978 & 11 & 24 & 43837.259 & $V=16.70$ \\
\hline 1971 & 10 & 20 & 41245.258 & $B=15.35$ & 1971 & 09 & 11 & 41206.363 & $\mathrm{I}=12.45$ & 1979 & 07 & 18 & 44072.527 & $\mathrm{I}=12.85$ \\
\hline 1972 & 07 & 06 & 41505.479 & $\mathrm{~B}=16.00$ & 1971 & 09 & 17 & 41212.409 & $\mathrm{I}=13.00$ & 1979 & 08 & 13 & 44099.420 & $V=16.60$ \\
\hline 1972 & 07 & 06 & 41505.493 & $V=14.55$ & 1971 & 09 & 17 & 41212.429 & $B=18.00$ & 1979 & 08 & 13 & 44099.442 & $\mathrm{I}=12.70$ \\
\hline 1972 & 05 & 12 & 41449.599 & $\mathrm{~B}=15.35$ & 1971 & 09 & 21 & 41216.377 & $\mathrm{~B}=18.20$ & 1979 & 08 & 21 & 44107.352 & $V=16.50$ \\
\hline 1972 & 06 & 18 & 41486.515 & $\mathrm{~B}=15.80$ & 1971 & 09 & 21 & 41216.399 & $\mathrm{I}=12.55$ & 1979 & 08 & 29 & 44115.400 & $V=16.90$ \\
\hline 1972 & 06 & 19 & 41487.554 & $V=16.40$ & 1971 & 10 & 08 & 41233.291 & $\mathrm{~B}=18.20$ & 1979 & 08 & 29 & 44115.425 & $\mathrm{I}=12.75$ \\
\hline 1972 & 08 & 04 & 41534.418 & $V=14.55$ & 1971 & 10 & 08 & 41233.311 & $\mathrm{I}=13.00$ & 1979 & 10 & 10 & 44157.313 & $V=16.90$ \\
\hline 1972 & 08 & 12 & 41542.427 & $V=14.45$ & 1971 & 10 & 20 & 41245.277 & $\mathrm{~B}=18.20$ & 1979 & 10 & 20 & 44167.306 & $V=17.00$ \\
\hline 1973 & 09 & 19 & 41945.324 & $\mathrm{~V}=14.35$ & 1971 & 10 & 20 & 41245.297 & $\mathrm{I}=13.10$ & 1979 & 10 & 29 & 44176.356 & $\mathrm{I}=13.20$ \\
\hline 1973 & 09 & 19 & 41945.327 & $\mathrm{~V}=14.40$ & 1971 & 11 & 06 & 41262.217 & $\mathrm{I}=13.00$ & 1980 & 05 & 13 & 44372.532 & $\mathrm{I}=13.00$ \\
\hline
\end{tabular}


Table 2. (continues)

\begin{tabular}{|c|c|c|c|c|c|c|c|c|c|c|c|c|c|c|}
\hline \multicolumn{3}{|c|}{ date } & HJD & mag & & late & & HJD & $\mathrm{mag}$ & & late & & HJD & mag \\
\hline \multicolumn{5}{|c|}{ Ap 3-1 } & \multicolumn{5}{|c|}{ CM Aql } & \multicolumn{5}{|c|}{ V443 Her } \\
\hline 1980 & 06 & 15 & 44406.459 & $B=18.00$ & 1961 & 09 & 09 & 37552.388 & $B=16.40$ & 1968 & 06 & 03 & 40010.514 & $\mathrm{~B}=12.85$ \\
\hline 1980 & 06 & 15 & 44406.485 & $\mathrm{I}=13.20$ & 1961 & 09 & 10 & 37553.392 & $B=16.40$ & 1968 & 06 & 17 & 40025.434 & $B=12.70$ \\
\hline 1980 & 09 & 04 & 44487.385 & $\mathrm{I}=13.00$ & 1961 & 09 & 13 & 37556.363 & $B>15.30$ & 1968 & 06 & 18 & 40026.449 & $B=12.70$ \\
\hline 1980 & 09 & 04 & 44487.408 & $\mathrm{~V}=17.00$ & 1961 & 09 & 15 & 37558.386 & $B>15.30$ & 1968 & 06 & 20 & 40028.422 & $B=12.55$ \\
\hline 1981 & 06 & 05 & 44760.535 & $\mathrm{I}=13.15$ & 1961 & 09 & 16 & 37559.373 & $\mathrm{~B}=16.40$ & 1968 & 06 & 21 & 40029.449 & $\mathrm{~B}=12.80$ \\
\hline 1981 & 06 & 05 & 44760.565 & $\mathrm{~V}=17.00$ & 1961 & 09 & 17 & 37560.419 & $B=16.50$ & 1968 & 06 & 22 & 40029.517 & $\mathrm{~B}=12.60$ \\
\hline 1982 & 05 & 28 & 45118.476 & $\mathrm{I}=13.10$ & 1961 & 10 & 02 & 37575.359 & $B=16.40$ & 1968 & 06 & 24 & 40032.446 & $\mathrm{~B}=12.70$ \\
\hline 1982 & 05 & 29 & 45118.512 & $\mathrm{~B}=18.00$ & 1961 & 10 & 05 & 37578.342 & $B=15.70$ & 1968 & 06 & 30 & 40038.410 & $B=12.60$ \\
\hline 1982 & 06 & 15 & 45135.512 & $V=17.00$ & 1961 & 10 & 09 & 37582.329 & $B=15.50$ & 1968 & 09 & 17 & 40117.323 & $B=12.60$ \\
\hline 1982 & 08 & 13 & 45194.510 & $\mathrm{I}=12.50$ & 1961 & 10 & 10 & 37583.342 & $B=16.05$ & 1972 & 08 & 08 & 41538.449 & $B=12.30$ \\
\hline 1982 & 08 & 23 & 45205.403 & $\mathrm{I}=12.85$ & 1962 & 07 & 23 & 37869.439 & $B=14.40$ & 1972 & 08 & 12 & 41542.435 & $\mathrm{~B}=12.30$ \\
\hline 1982 & 09 & 17 & 45230.364 & $\mathrm{I}=12.85$ & 1962 & 08 & 03 & 37880.500 & $B=14.60$ & 1972 & 08 & 12 & 41542.456 & $\mathrm{~B}=12.40$ \\
\hline 1982 & 09 & 17 & 45230.392 & $\mathrm{~V}=16.80$ & 1963 & 07 & 19 & 38230.474 & $B=15.50$ & 1972 & 08 & 12 & 41542.474 & $\mathrm{~B}=12.35$ \\
\hline 1983 & 06 & 15 & 45501.410 & $\mathrm{I}=13.00$ & 1964 & 09 & 07 & 38646.415 & $B=15.60$ & 1972 & 08 & 13 & 41543.403 & $\mathrm{~B}=12.35$ \\
\hline 1983 & 06 & 15 & 45501.438 & $\mathrm{~V}=16.70$ & 1965 & 10 & 24 & 39058.276 & $B=13.70$ & 1972 & 08 & 13 & 41543.419 & $B=12.30$ \\
\hline 1983 & 07 & 02 & 45518.460 & $\mathrm{I}=12.85$ & 1966 & 07 & 20 & 39326.527 & $B=14.70$ & 1972 & 08 & 13 & 41543.440 & $\mathrm{~B}=12.30$ \\
\hline 1983 & 08 & 04 & 45551.411 & $\mathrm{I}=12.80$ & 1982 & 09 & 15 & 45228.366 & $\mathrm{~V}=13.50$ & 1972 & 08 & 15 & 41545.440 & $\mathrm{~B}=12.25$ \\
\hline 1983 & 08 & 11 & 45558.446 & $V=16.90$ & \multirow{3}{*}{\multicolumn{5}{|c|}{ V1413 Aql }} & 1972 & 08 & 15 & 41545.454 & $\mathrm{~B}=12.25$ \\
\hline 1983 & 09 & 05 & 45583.460 & $\mathrm{~V}=17.00$ & & & & & & 1972 & 08 & 15 & 41545.468 & $\mathrm{~B}=12.40$ \\
\hline 1983 & 09 & 29 & 45607.331 & $\mathrm{I}=13.50$ & & & & & & 1972 & 08 & 16 & 41546.436 & $B=12.40$ \\
\hline 1984 & 07 & 28 & 45909.531 & $\mathrm{I}=13.50$ & 1962 & 03 & 15 & 37738.667 & $B=14.10$ & 1972 & 08 & 16 & 41546.466 & $B=12.40$ \\
\hline 1984 & 08 & 03 & 45916.434 & $\mathrm{I}=12.90$ & 1962 & 05 & 01 & 37787.602 & $B=14.80$ & 1972 & 08 & 16 & 41546.481 & $\mathrm{~B}=12.35$ \\
\hline 1985 & 08 & 12 & 46290.403 & $\mathrm{I}=12.60$ & 1963 & 10 & 11 & 38314.286 & $B=15.60$ & 1972 & 09 & 28 & 41589.308 & $\mathrm{~B}=12.40$ \\
\hline 1985 & 08 & 12 & 46290.430 & $\mathrm{~V}=16.95$ & 1963 & 10 & 12 & 38315.284 & $B=15.60$ & 1972 & 09 & 29 & 41590.369 & $\mathrm{~B}=12.40$ \\
\hline 1985 & 08 & 21 & 46299.379 & $\mathrm{I}=13.00$ & 1963 & 10 & 16 & 38319.294 & $B=15.70$ & 1972 & 11 & 03 & 41625.317 & $\mathrm{~B}=12.35$ \\
\hline 1985 & 09 & 06 & 46315.348 & $\mathrm{I}=13.40$ & 1963 & 10 & 19 & 38322.260 & $\mathrm{~B}=15$ & 1972 & 11 & 06 & 41628.250 & $\mathrm{~B}=12.40$ \\
\hline 1986 & 10 & 28 & 46732.307 & $\mathrm{I}=12.50$ & 1963 & 10 & 21 & 38324.289 & $B=15.65$ & 1972 & 11 & 08 & 41630.289 & $\mathrm{~B}=12.35$ \\
\hline 1988 & 08 & 07 & 47381.410 & $\mathrm{I}=13.25$ & 1963 & 11 & 07 & 38341.247 & $\mathrm{~B}=15.95$ & 1973 & 05 & 26 & 41828.559 & $\mathrm{~B}=12.60$ \\
\hline 1988 & 08 & 07 & 47381.443 & $\mathrm{~V}=16.70$ & 1963 & 11 & 17 & 38351.266 & $B=16.10$ & 1973 & 06 & 29 & 41863.424 & $\mathrm{~B}=12.60$ \\
\hline 1988 & 08 & 10 & 47384.469 & $\mathrm{I}=13.30$ & 1964 & 11 & 05 & 38705.246 & $B=15.00$ & 1973 & 06 & 29 & 41863.443 & $\mathrm{~V}=11.45$ \\
\hline 1988 & 09 & 07 & 47412.431 & $\mathrm{~V}=16.80$ & 1965 & 10 & 23 & 39057.261 & $B=14.55$ & 1973 & 07 & 03 & 41867.433 & $B=12.60$ \\
\hline 1988 & 09 & 07 & 47412.450 & $\mathrm{I}=13.00$ & 1966 & 07 & 16 & 39323.469 & $\mathrm{~B}=16.15$ & 1973 & 07 & 03 & 41867.469 & $\mathrm{~B}=12.60$ \\
\hline 1988 & 09 & 17 & 47422.410 & $\mathrm{I}=12.85$ & 1966 & 09 & 22 & 39391.457 & $\mathrm{~B}=15.80$ & 1973 & 07 & 04 & 41867.506 & $\mathrm{~B}=12.60$ \\
\hline 1988 & 09 & 18 & 47423.395 & $V=16.50$ & 1967 & 06 & 30 & 39672.466 & $B=15.80$ & 1973 & 07 & 21 & 41885.403 & $\mathrm{~B}=12.50$ \\
\hline 1990 & 08 & 21 & 48125.352 & $\mathrm{~V}=16.30$ & 1967 & 07 & 04 & 39676.449 & $B=16.50$ & 1973 & 07 & 22 & 41886.420 & $\mathrm{~B}=12.75$ \\
\hline 1990 & 08 & 21 & 48125.378 & $\mathrm{I}=12.9$ & 1967 & 10 & 21 & 39784.420 & $\mathrm{~B}=15$ & 1973 & 07 & 22 & 41886.434 & $B=12.50$ \\
\hline 1992 & 07 & 03 & 48807.492 & $\mathrm{~V}=16.30$ & 1967 & 10 & 22 & 39784.398 & $B=15.30$ & 1973 & 07 & 25 & 41889.457 & $B=12.50$ \\
\hline 1992 & 07 & 04 & 48807.519 & $\mathrm{I}=12.65$ & 1967 & 11 & 24 & 39819.223 & $\mathrm{~V}=14.40$ & 1973 & 07 & 26 & 41889.518 & $B=12.60$ \\
\hline 1992 & 08 & 02 & 48836.516 & $\mathrm{I}=12.30$ & 1967 & 11 & 24 & 39819.238 & $\mathrm{~B}=14.80$ & 1973 & 07 & 26 & 41890.476 & $B=12.65$ \\
\hline 1992 & 09 & 02 & 48868.379 & $\mathrm{I}=13.10$ & 1968 & 07 & 22 & 40060.433 & $B=14.00$ & 1973 & 07 & 27 & 41890.507 & $B=12.60$ \\
\hline 1992 & 09 & 02 & 48868.406 & $B=18.40$ & 1968 & 08 & 24 & 40093.379 & $B=14.50$ & 1973 & 07 & 27 & 41890.535 & $B=12.60$ \\
\hline 1993 & 05 & 21 & 49129.486 & $\mathrm{I}=13.10$ & 1968 & 09 & 11 & 40111.394 & $\mathrm{~V}=14.30$ & 1973 & 07 & 28 & 41892.436 & $\mathrm{~B}=12.80$ \\
\hline 1993 & 05 & 22 & 49129.515 & $\mathrm{~V}=16.90$ & 1968 & 09 & 24 & 40124.381 & $B=15.70$ & 1973 & 07 & 28 & 41892.461 & $B=12.50$ \\
\hline 1993 & 07 & 14 & 49182.521 & $B=18.60$ & 1968 & 11 & 22 & 40183.231 & $B=15.80$ & 1973 & 08 & 05 & 41900.424 & $B=12.65$ \\
\hline 1993 & 07 & 14 & 49182.549 & $\mathrm{I}=12.75$ & 1969 & 08 & 17 & 40451.356 & $\mathrm{~V}=14.70$ & 1973 & 10 & 19 & 41975.248 & $\mathrm{~B}=12.45$ \\
\hline 1993 & 08 & 15 & 49215.430 & $\mathrm{I}=13.20$ & 1969 & 09 & 11 & 40476.410 & $B=15.60$ & 1973 & 10 & 20 & 41976.254 & $B=12.40$ \\
\hline 1994 & 08 & 12 & 49577.476 & $\mathrm{~V}=17.00$ & 1969 & 10 & 29 & 40524.227 & $B=13.15$ & 1974 & 05 & 27 & 42194.579 & $B=12.50$ \\
\hline 1995 & 05 & 05 & 49842.517 & $\mathrm{I}=13.2$ & 1969 & 11 & 01 & 40527.259 & $\mathrm{~V}=12$ & 1974 & 06 & 13 & 42212.452 & $B=12.50$ \\
\hline 1995 & 05 & 05 & 49842.539 & $\mathrm{~V}=16.9$ & 1969 & 11 & 28 & 40554.208 & $B=15$ & 1974 & 06 & 15 & 42214.458 & $B=12.50$ \\
\hline 1995 & 06 & 25 & 49894.488 & $\mathrm{~V}=15.90$ & 1970 & 07 & 06 & 40773.517 & $\mathrm{~V}=13.50$ & 1974 & 07 & 15 & 42244.486 & $B=12.55$ \\
\hline 1995 & 09 & 26 & 49987.368 & $V=15.90$ & 1970 & 09 & 09 & 40839.403 & $B=14.05$ & 1974 & 07 & 16 & 42244.500 & $\mathrm{~B}=12.45$ \\
\hline \multirow{3}{*}{\multicolumn{5}{|c|}{ CM Aql }} & 1970 & 10 & 26 & 40886.321 & $\mathrm{~V}=13.70$ & 1974 & 07 & 16 & 42244.514 & $\mathrm{~B}=12.50$ \\
\hline & & & & & 1970 & 10 & 26 & 40886.334 & $\mathrm{~B}=14.25$ & 1974 & 07 & 19 & 42248.437 & $\mathrm{~B}=12.50$ \\
\hline & & & & & 1971 & 03 & 31 & 41041.575 & $B=12.55$ & 1974 & 07 & 21 & 42250.467 & $B=12.45$ \\
\hline 1961 & 06 & 15 & 37466.491 & $B=16.20$ & 1971 & 03 & 31 & 41041.591 & $\mathrm{~V}=12.00$ & 1974 & 07 & 21 & 42250.484 & $B=12.55$ \\
\hline 1961 & 07 & 09 & 37489.511 & $B=16.10$ & 1971 & 08 & 29 & 41193.433 & $\mathrm{~V}=12.00$ & 1974 & 07 & 23 & 42251.540 & $\mathrm{~B}=12.50$ \\
\hline 1961 & 07 & 14 & 37494.535 & $B=16.40$ & 1971 & 10 & 20 & 41245.283 & $\mathrm{~V}=12.00$ & 1974 & 08 & 13 & 42273.459 & $\mathrm{~B}=12.60$ \\
\hline 1961 & 07 & 15 & 37495.514 & $B=16.40$ & 1974 & 09 & 13 & 42304.348 & $B=15.60$ & 1974 & 08 & 13 & 42273.474 & $B=12.50$ \\
\hline 1961 & 08 & 02 & 37514.422 & $B>15.30$ & 1981 & 06 & 29 & 44785.463 & $B=14.90$ & 1974 & 08 & 15 & 42275.409 & $B=12.60$ \\
\hline 1961 & 08 & 09 & 37521.407 & $B=16.40$ & 1993 & 05 & 25 & 49133.438 & $\mathrm{~V}=13.70$ & 1974 & 08 & 15 & 42275.423 & $B=12.60$ \\
\hline 1961 & 08 & 10 & 37522.427 & $B=15.80$ & 1993 & 07 & 07 & 49175.525 & $B=14.50$ & 1974 & 08 & 17 & 42277.447 & $B=12.60$ \\
\hline 1961 & 08 & 11 & 37523.484 & $B=16.40$ & 1993 & 07 & 07 & 49175.538 & $\mathrm{~V}=14.30$ & 1974 & 08 & 17 & 42277.461 & $\mathrm{~B}=12.70$ \\
\hline 1961 & 08 & 18 & 37530.464 & $B=16.40$ & 1993 & 08 & 12 & 49212.379 & $B=15.30$ & 1974 & 08 & 20 & 42280.410 & $\mathrm{~B}=12.50$ \\
\hline 1961 & 08 & 30 & 37542.385 & $B=16.40$ & 1993 & 08 & 12 & 49212.408 & $\mathrm{~V}=14.00$ & 1974 & 08 & 20 & 42280.424 & $B=12.60$ \\
\hline 1961 & 09 & 03 & 37546.354 & $\mathrm{~B}=16.30$ & 1993 & 08 & 19 & 49219.453 & $\mathrm{~V}=14.40$ & 1974 & 09 & 10 & 42301.365 & $\mathrm{~B}=12.55$ \\
\hline 1961 & 09 & 06 & 37549.402 & $B=16.40$ & 1993 & 11 & 16 & 49308.345 & $B=12.70$ & 1974 & 09 & 12 & 42303.447 & $B=12.70$ \\
\hline
\end{tabular}


Table 2. (continues)

\begin{tabular}{|c|c|c|c|c|c|c|c|c|c|c|c|c|c|c|}
\hline \multicolumn{3}{|c|}{ date } & HJD & mag & & late & & HJD & mag & & date & & HJD & mag \\
\hline \multicolumn{5}{|c|}{ V443 Her } & \multicolumn{5}{|c|}{ V627 Cas } & \multicolumn{5}{|c|}{ V627 Cas } \\
\hline 1974 & 09 & 13 & 42304.365 & $B=12.65$ & 1971 & 12 & 11 & 41297.247 & $\mathrm{I}=8.85$ & 1980 & 11 & 09 & 44552.502 & $\mathrm{I}=9.60$ \\
\hline 1974 & 09 & 14 & 42305.330 & $\mathrm{~B}=12.80$ & 1971 & 12 & 11 & 41297.276 & $B=14.50$ & 1980 & 11 & 13 & 44557.427 & $\mathrm{~B}=15.85$ \\
\hline 1974 & 09 & 14 & 42305.345 & $B=12.90$ & 1971 & 12 & 14 & 41300.208 & $\mathrm{I}=8.80$ & 1980 & 11 & 13 & 44557.454 & $\mathrm{I}=9.60$ \\
\hline 1974 & 09 & 14 & 42305.359 & $\mathrm{~B}=12.80$ & 1971 & 12 & 14 & 41300.236 & $B=14.50$ & 1981 & 09 & 06 & 44854.403 & $\mathrm{I}=9.60$ \\
\hline 1974 & 09 & 16 & 42307.412 & $\mathrm{~B}=12.80$ & 1972 & 09 & 09 & 41570.448 & $B=15.00$ & 1981 & 10 & 27 & 44905.459 & $B=15.60$ \\
\hline 1974 & 10 & 09 & 42330.416 & $B=12.55$ & 1972 & 09 & 09 & 41570.474 & $\mathrm{I}=9.35$ & 1981 & 11 & 02 & 44911.348 & $\mathrm{I}=9.60$ \\
\hline 1974 & 10 & 17 & 42338.303 & $B=12.60$ & 1972 & 10 & 15 & 41606.400 & $\mathrm{I}=9.35$ & 1981 & 11 & 02 & 44911.377 & $B=15.60$ \\
\hline 1975 & 06 & 04 & 42568.427 & $B=12.60$ & 1972 & 10 & 15 & 41606.420 & $B=14.90$ & 1981 & 11 & 24 & 44933.410 & $B=15.55$ \\
\hline 1975 & 06 & 13 & 42577.461 & $B=12.55$ & 1972 & 10 & 31 & 41622.398 & $B=14.90$ & 1981 & 11 & 24 & 44933.441 & $\mathrm{I}=9.35$ \\
\hline 1975 & 07 & 02 & 42596.480 & $\mathrm{~B}=12.80$ & 1972 & 10 & 31 & 41622.421 & $\mathrm{I}=8.80$ & 1982 & 10 & 21 & 45264.323 & $\mathrm{I}=9.60$ \\
\hline 1975 & 08 & 09 & 42634.440 & $B=12.50$ & 1972 & 11 & 04 & 41626.341 & $B=14.80$ & 1982 & 10 & 21 & 45264.353 & $V=12.60$ \\
\hline 1975 & 08 & 13 & 42638.430 & $B=12.30$ & 1972 & 11 & 04 & 41626.367 & $\mathrm{I}=9.10$ & 1982 & 11 & 16 & 45290.377 & $B=15.65$ \\
\hline 1975 & 09 & 03 & 42659.430 & $B=12.40$ & 1972 & 11 & 27 & 41649.225 & $\mathrm{I}=9.60$ & 1983 & 09 & 13 & 45591.417 & $\mathrm{I}=9.60$ \\
\hline 1975 & 09 & 12 & 42668.497 & $B=12.30$ & 1972 & 11 & 27 & 41649.246 & $B=14.80$ & 1983 & 09 & 13 & 45591.442 & $\mathrm{~V}=12.80$ \\
\hline 1975 & 10 & 02 & 42688.392 & $B=12.45$ & 1972 & 12 & 07 & 41659.283 & $V=11.85$ & 1983 & 10 & 03 & 45611.462 & $B=15.60$ \\
\hline 1975 & 10 & 05 & 42691.353 & $B=12.50$ & 1972 & 12 & 07 & 41659.302 & $\mathrm{I}=9.10$ & 1993 & 08 & 23 & 49223.387 & $R=11.90$ \\
\hline 1975 & 10 & 06 & 42692.357 & $B=12.40$ & 1972 & 12 & 13 & 41665.457 & $\mathrm{I}=9.35$ & & & & & \\
\hline 1975 & 10 & 26 & 42712.268 & $\mathrm{~B}=12.15$ & 1972 & 12 & 13 & 41665.477 & $\mathrm{~V}=12.05$ & \multicolumn{5}{|c|}{ V919 Sgr } \\
\hline 1975 & 10 & 27 & 42713.268 & $B=12.25$ & 1972 & 12 & 23 & 41675.213 & $\mathrm{I}=9.35$ & & & & & \\
\hline 1975 & 10 & 28 & 42714.270 & $B=12.30$ & 1972 & 12 & 23 & 41675.233 & $V=12.50$ & 1961 & 06 & 19 & 37469.561 & $\mathrm{~B}=13.80$ \\
\hline 1975 & 10 & 29 & 42715.367 & $B=12.25$ & 1973 & 10 & 03 & 41958.548 & $\mathrm{I}=9.80$ & 1967 & 06 & 30 & 39672.486 & $B=13.90$ \\
\hline 1975 & 10 & 31 & 42717.368 & $B=12.25$ & 1973 & 10 & 03 & 41958.571 & $B=14.80$ & 1967 & 07 & 01 & 39672.517 & $\mathrm{I}=10.20$ \\
\hline 1976 & 04 & 09 & 42877.618 & $\mathrm{~B}=12.30$ & 1973 & 10 & 20 & 41976.424 & $V=12.55$ & 1967 & 07 & 27 & 39699.432 & $\mathrm{I}=10.20$ \\
\hline 1976 & 06 & 02 & 42932.409 & $B=12.50$ & 1973 & 10 & 20 & 41976.464 & $B=15.00$ & 1967 & 07 & 28 & 39700.413 & $B=13.95$ \\
\hline 1976 & 06 & 19 & 42949.463 & $B=12.50$ & 1973 & 10 & 24 & 41980.473 & $\mathrm{I}=9.80$ & 1967 & 07 & 28 & 39700.429 & $\mathrm{I}=10.15$ \\
\hline 1976 & 06 & 28 & 42958.479 & $B=12.50$ & 1973 & 10 & 24 & 41980.496 & $B=14.95$ & 1967 & 07 & 29 & 39701.431 & $\mathrm{I}=10.20$ \\
\hline 1976 & 06 & 29 & 42959.451 & $B=12.50$ & 1973 & 10 & 27 & 41983.382 & $B=14.75$ & 1967 & 07 & 29 & 39701.458 & $\mathrm{~B}=13.95$ \\
\hline 1976 & 07 & 23 & 42983.424 & $B=12.80$ & 1973 & 10 & 27 & 41983.401 & $V=12.50$ & 1967 & 07 & 31 & 39703.397 & $\mathrm{I}=10.10$ \\
\hline 1976 & 08 & 01 & 42992.420 & $B=12.60$ & 1973 & 10 & 31 & 41987.464 & $B=14.70$ & 1967 & 07 & 31 & 39703.424 & $\mathrm{~B}=14.20$ \\
\hline 1976 & 08 & 23 & 43014.380 & $B=12.60$ & 1973 & 10 & 31 & 41987.485 & $\mathrm{I}=9.60$ & 1967 & 08 & 03 & 39706.453 & $\mathrm{I}=10.00$ \\
\hline 1976 & 09 & 18 & 43040.351 & $B=12.50$ & 1973 & 11 & 15 & 42002.315 & $V=12.60$ & 1967 & 09 & 06 & 39740.419 & $B=13.90$ \\
\hline 1977 & 05 & 25 & 43288.586 & $B=12.40$ & 1973 & 11 & 30 & 42017.394 & $\mathrm{~V}=12.60$ & 1967 & 09 & 27 & 39761.323 & $B=13.90$ \\
\hline 1977 & 08 & 19 & 43375.433 & $B=12.50$ & 1973 & 11 & 30 & 42017.422 & $\mathrm{I}=9.60$ & 1967 & 09 & 29 & 39763.313 & $\mathrm{I}=10.15$ \\
\hline 1979 & 05 & 24 & 44017.590 & $V=11.45$ & 1973 & 12 & 12 & 42029.242 & $V=12.60$ & 1967 & 10 & 05 & 39769.268 & $\mathrm{I}=10.20$ \\
\hline 1982 & 09 & 18 & 45231.318 & $B=12.30$ & 1973 & 12 & 12 & 42029.267 & $\mathrm{I}=9.60$ & 1967 & 10 & 05 & 39769.298 & $B=14.10$ \\
\hline 1983 & 06 & 03 & 45489.501 & $B=12.40$ & 1973 & 12 & 17 & 42034.306 & $\mathrm{I}=9.60$ & 1968 & 07 & 06 & 40043.514 & $\mathrm{~B}=14.20$ \\
\hline 1993 & 08 & 16 & 49216.468 & $\mathrm{I}=8.80$ & 1973 & 12 & 17 & 42034.327 & $V=12.60$ & 1968 & 07 & 06 & 40043.533 & $\mathrm{I}=10.10$ \\
\hline & & & & & 1975 & 10 & 03 & 42689.367 & $B=14.80$ & 1968 & 07 & 20 & 40058.450 & $\mathrm{I}=10.10$ \\
\hline & & & 627 Cas & & 1975 & 10 & 03 & 42689.389 & $\mathrm{I}=9.60$ & 1968 & 07 & 20 & 40058.470 & $\mathrm{~B}=14.20$ \\
\hline & & & & & 1975 & 10 & 26 & 42712.388 & $B=14.65$ & 1968 & 07 & 22 & 40060.378 & $\mathrm{I}=10.00$ \\
\hline 1969 & 11 & 03 & 40529.262 & $B=14.70$ & 1975 & 10 & 26 & 42712.416 & $\mathrm{I}=9.60$ & 1968 & 07 & 22 & 40060.395 & $B=14.30$ \\
\hline 1969 & 11 & 03 & 40529.286 & $\mathrm{I}=8.80$ & 1975 & 11 & 29 & 42746.314 & $B=14.85$ & 1968 & 09 & 18 & 40118.390 & $\mathrm{I}=10.15$ \\
\hline 1970 & 09 & 06 & 40836.433 & $B=14.75$ & 1975 & 11 & 29 & 42746.336 & $\mathrm{I}=9.80$ & 1968 & 09 & 24 & 40124.356 & $B=14.40$ \\
\hline 1970 & 09 & 06 & 40836.460 & $\mathrm{I}=8.80$ & 1976 & 09 & 17 & 43039.468 & $B=14.90$ & 1968 & 09 & 24 & 40124.376 & $\mathrm{I}=10.25$ \\
\hline 1970 & 10 & 22 & 40882.441 & $B=14.70$ & 1976 & 09 & 17 & 43039.489 & $\mathrm{I}=8.85$ & 1968 & 10 & 19 & 40149.257 & $\mathrm{I}=10.25$ \\
\hline 1970 & 10 & 22 & 40882.465 & $\mathrm{I}=8.80$ & 1976 & 10 & 22 & 43074.316 & $\mathrm{I}=8.80$ & 1968 & 10 & 19 & 40149.269 & $\mathrm{~B}=14.50$ \\
\hline 1970 & 10 & 25 & 40885.418 & $B=14.70$ & 1976 & 10 & 22 & 43074.341 & $B=14.80$ & 1968 & 10 & 24 & 40154.256 & $\mathrm{I}=10.10$ \\
\hline 1970 & 10 & 28 & 40888.485 & $\mathrm{I}=9.35$ & 1976 & 12 & 14 & 43127.397 & $B=14.90$ & 1968 & 10 & 24 & 40154.267 & $\mathrm{~B}=14.40$ \\
\hline 1970 & 10 & 29 & 40888.512 & $B=14.75$ & 1976 & 12 & 14 & 43127.421 & $\mathrm{I}=8.85$ & 1969 & 05 & 23 & 40364.594 & $\mathrm{I}=9.90$ \\
\hline 1970 & 11 & 04 & 40895.430 & $R=11.30$ & 1977 & 09 & 05 & 43392.472 & $\mathrm{I}=9.35$ & 1969 & 05 & 27 & 40368.563 & $\mathrm{I}=10.10$ \\
\hline 1970 & 11 & 04 & 40895.457 & $B=14.50$ & 1977 & 09 & 05 & 43392.494 & $B=15.50$ & 1969 & 05 & 27 & 40368.577 & $B=14.45$ \\
\hline 1970 & 11 & 27 & 40918.234 & $\mathrm{I}=8.85$ & 1977 & 09 & 11 & 43398.460 & $\mathrm{I}=9.10$ & 1969 & 07 & 03 & 40405.521 & $B=14.15$ \\
\hline 1970 & 11 & 27 & 40918.259 & $B=14.55$ & 1977 & 09 & 11 & 43398.485 & $B=15.50$ & 1969 & 07 & 10 & 40413.491 & $B=14.10$ \\
\hline 1970 & 12 & 03 & 40924.317 & $\mathrm{I}=8.80$ & 1977 & 09 & 18 & 43405.431 & $B=15.55$ & 1969 & 07 & 14 & 40416.537 & $\mathrm{I}=10.20$ \\
\hline 1970 & 12 & 03 & 40924.342 & $B=14.75$ & 1977 & 09 & 18 & 43405.462 & $\mathrm{I}=9.60$ & 1969 & 07 & 15 & 40418.398 & $B=14.40$ \\
\hline 1970 & 12 & 20 & 40941.258 & $B=14.75$ & 1977 & 10 & 03 & 43420.403 & $\mathrm{I}=9.35$ & 1969 & 07 & 15 & 40418.416 & $\mathrm{I}=10.10$ \\
\hline 1970 & 12 & 20 & 40941.286 & $\mathrm{I}=8.85$ & 1977 & 11 & 04 & 43452.278 & $\mathrm{I}=9.35$ & 1969 & 08 & 14 & 40448.436 & $\mathrm{I}=10.10$ \\
\hline 1971 & 01 & 18 & 40970.230 & $\mathrm{I}=8.85$ & 1977 & 11 & 16 & 43464.351 & $\mathrm{I}=9.10$ & 1969 & 08 & 16 & 40450.421 & $B=14.00$ \\
\hline 1971 & 08 & 15 & 41179.475 & $B=14.60$ & 1977 & 12 & 02 & 43480.354 & $B=15.40$ & 1969 & 09 & 15 & 40480.400 & $\mathrm{~B}=14.00$ \\
\hline 1971 & 08 & 16 & 41179.503 & $R=11.30$ & 1977 & 12 & 02 & 43480.439 & $\mathrm{I}=9.35$ & 1969 & 09 & 15 & 40480.418 & $\mathrm{I}=10.10$ \\
\hline 1971 & 08 & 16 & 41179.532 & $\mathrm{I}=9.80$ & 1977 & 12 & 14 & 43492.303 & $\mathrm{I}=9.35$ & 1969 & 10 & 09 & 40504.305 & $\mathrm{I}=10.15$ \\
\hline 1971 & 09 & 20 & 41215.478 & $B=14.50$ & 1977 & 12 & 14 & 43492.381 & $B=15.60$ & 1969 & 10 & 09 & 40504.321 & $B=14.35$ \\
\hline 1971 & 09 & 21 & 41215.509 & $\mathrm{I}=8.90$ & 1978 & 10 & 07 & 43789.409 & $V=12.60$ & 1969 & 10 & 10 & 40505.337 & $\mathrm{I}=10.20$ \\
\hline 1971 & 10 & 20 & 41245.403 & $B=14.60$ & 1978 & 10 & 07 & 43789.433 & $\mathrm{I}=9.60$ & 1969 & 10 & 13 & 40508.290 & $\mathrm{I}=10.20$ \\
\hline 1971 & 10 & 20 & 41245.423 & $\mathrm{I}=8.80$ & 1979 & 08 & 30 & 44116.380 & $\mathrm{I}=9.60$ & 1969 & 10 & 13 & 40508.306 & $B=14.00$ \\
\hline 1971 & 11 & 16 & 41272.393 & $B=14.60$ & 1979 & 08 & 30 & 44116.495 & $B=15.60$ & 1969 & 10 & 16 & 40511.257 & $\mathrm{I}=10.20$ \\
\hline 1971 & 11 & 16 & 41272.425 & $\mathrm{I}=8.80$ & 1979 & 09 & 26 & 44143.443 & $\mathrm{I}=9.60$ & 1970 & 08 & 02 & 40801.453 & $\mathrm{I}=10.20$ \\
\hline 1971 & 12 & 05 & 41291.206 & $\mathrm{I}=8.80$ & 1979 & 09 & 26 & 44143.471 & $B=15.60$ & 1970 & 08 & 02 & 40801.467 & $B=14.30$ \\
\hline 1971 & 12 & 05 & 41291.230 & $B=14.65$ & 1979 & 10 & 25 & 44172.450 & $B=15.75$ & 1970 & 08 & 06 & 40805.447 & $B=14.20$ \\
\hline 1971 & 12 & 06 & 41292.213 & $\mathrm{I}=8.85$ & 1979 & 10 & 25 & 44172.471 & $\mathrm{I}=9.60$ & 1970 & 08 & 06 & 40805.457 & $\mathrm{I}=10.15$ \\
\hline 1971 & 12 & 06 & 41292.238 & $B=14.65$ & 1980 & 11 & 08 & 44552.478 & $V=12.60$ & & & & & \\
\hline
\end{tabular}


against the $U B V(R I)_{\mathrm{C}}$ comparison sequences calibrated by Henden and Munari (2000). These comparison sequences proved to work perfectly, covering the range of variability of the program stars and with both the comparison stars and the variable visible at the same time in the eyepiece field of the microscope. The exception has been V627 Cas, which was brighter than the comparison sequence in some of the $R$ and $I$ plates. We then searched outside the field explored by Henden and Munari (2000) for bright stars that have been found constant in brightness by Hipparcos/Tycho. We converted their Tycho $B_{T}$, $V_{T}$ magnitudes into Johnson's standard $B$ and $V$ values, and using the transformations of Caldwell et al. (1993) we eventually derived their $R_{\mathrm{C}}$ and $I_{\mathrm{C}}$ magnitudes. These transformation relations between colors in the $U B V(R I)_{\mathrm{C}}$ system gives accurate results provided that the stars belong to the solar neighborhood population, the reddening is not large and the luminosity class is roughly known. We have assumed all the selected Tycho objects to be nearby main sequence stars. Thus, the $R_{\mathrm{C}}$ and $I_{\mathrm{C}}$ so derived may be considered only as guidelines useful for estimating photographic plates. The two stars we used to extend the $R_{\mathrm{C}}$ and $I_{\mathrm{C}}$ comparison sequences around V627 Cas are TYC $399722031\left(R_{\mathrm{C}}=9.11\right.$ and $\left.I_{\mathrm{C}}=9.10\right)$ and TYC $399718681\left(R_{\mathrm{C}}=8.71\right.$ and $\left.I_{\mathrm{C}}=8.67\right)$.

The data are presented in Table 1 . The date (year/ month/ day/ format), the heliocentric JD and the estimated magnitude (in steps of $0.05 \mathrm{mag}$ ) are given. Further details (including plate number, exposure time, emulsion and filter types, etc.) are available via http://ulisse.pd.astro.it/symbio_pg/.

\section{Notes on individual objects}

Brief notes follow to comment upon the photometric behavior displayed by the program stars.

Ap 3-1 varied by $\triangle I \sim \triangle B \sim 1$ mag over the 26 years covered by the Asiago plates in Table 1, but without following any obvious periodic pattern or monotonic trend.

V1413 Aql. The 1962-1981 sub-set of plates were already analyzed by Munari (1992) against a different comparison sequence. The Table 1 data confirm the preoutburst lightcurve modulated by a reflection effect following the 434.1 day orbital period of the post-outburst eclipses.

V443 Her. 96 of the $100 B$ band data in Table 1 cover the period 1968-1977. They confirm in periodicity (594 day) and amplitude ( $\triangle B \sim 0.4 \mathrm{mag})$ the later 1979-1993 $B$ lightcurve of Kolotilov et al. (1995). Unavoidable small differences in pass-band profiles between the photoelectric and photographic realization of the $B$ band can contribute to the slight difference in mean brightness $(B=12.43$ for Kolotilov et al. photoelectric photometry, $B=12.53$ for Table 1 data).

V919 Sgr. Our 1961-1970 data are affected only by a small amplitude variability: $\triangle I \sim 0.3$ and $\triangle B \sim 0.5$ mag.
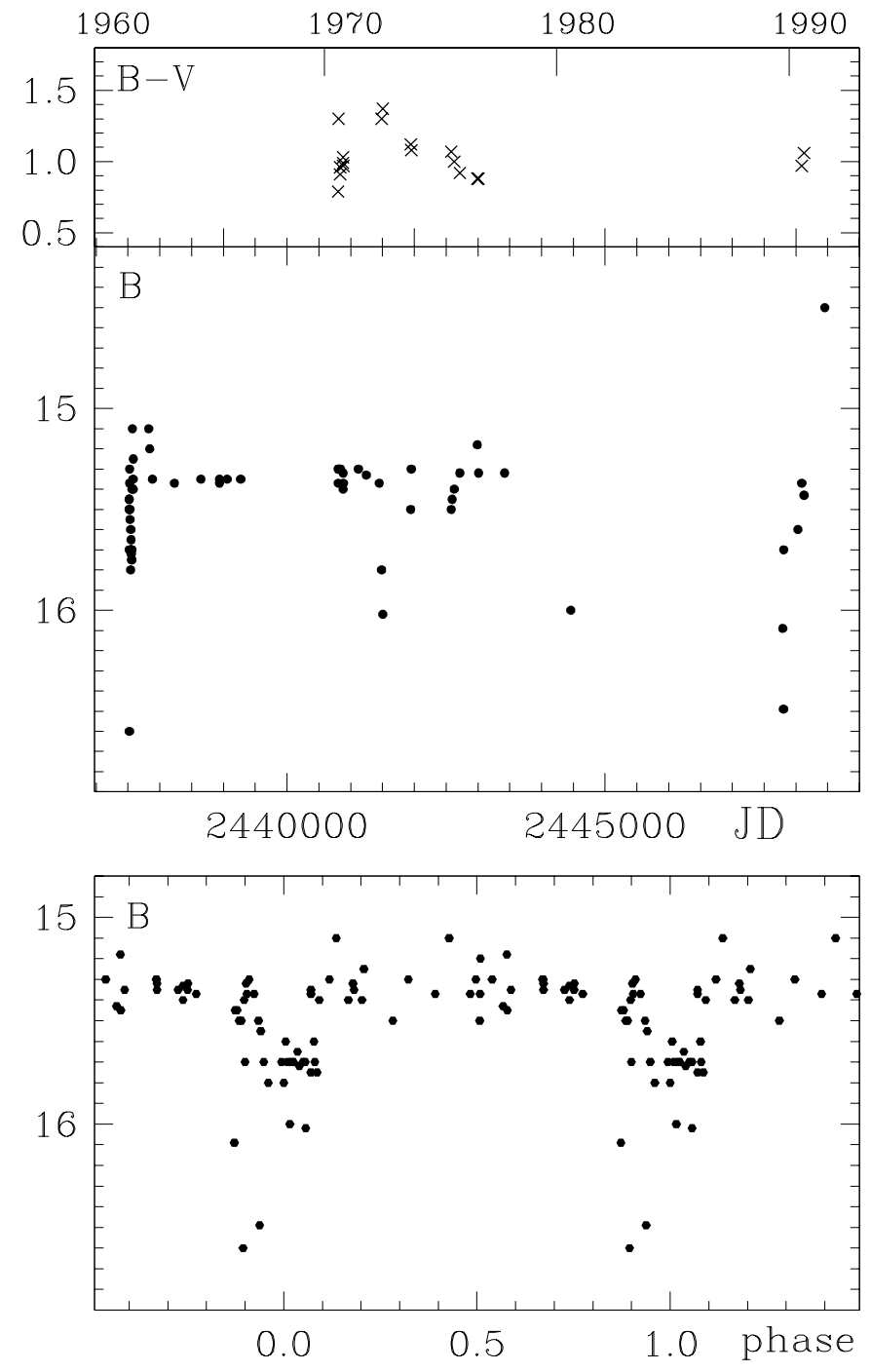

Fig. 1. $B$ and $B-V$ lightcurves of AS 323 (upper panels). The $B$ data folded onto a $\mathrm{P}=197.6$ day period (lower panel).

\section{1. $A S 323$}

Originally classified as a planetary nebula $(=\mathrm{K} 4-7=$ PK $26-2^{\circ} 2$ ), the symbiotic nature of AS 323 has been discovered by Sabbadin (1986) and Acker et al. (1988). The spectrum resembles a proto-type symbiotic star, with well developed $\mathrm{TiO}$ bands in the red, veiling by the circumstellar nebula in the blue and a high ionization emission line spectrum (He II $4686 \AA$ and $6825 \AA$ Raman scattering of O VI are both prominent), with weak or absent nebular lines. Mikolajewska et al. (1997) estimated a M3 spectral type for the cool giant and $T_{\text {eff }} \geq 100000 \mathrm{~K}$ and $L=1200 L_{\odot}$ for the hot companion. Munari et al. (2001) report $B=15.18, B-V=+0.99, U-B=-0.42$, $V-R_{C}=+1.11$ and $R_{C}-I_{C}=+1.39$ for observations obtained in 1999 .

The AS 323 data from Table 1 are plotted in Figure 1. The 1960-1990 lightcurve is characterized by a flat quies- 


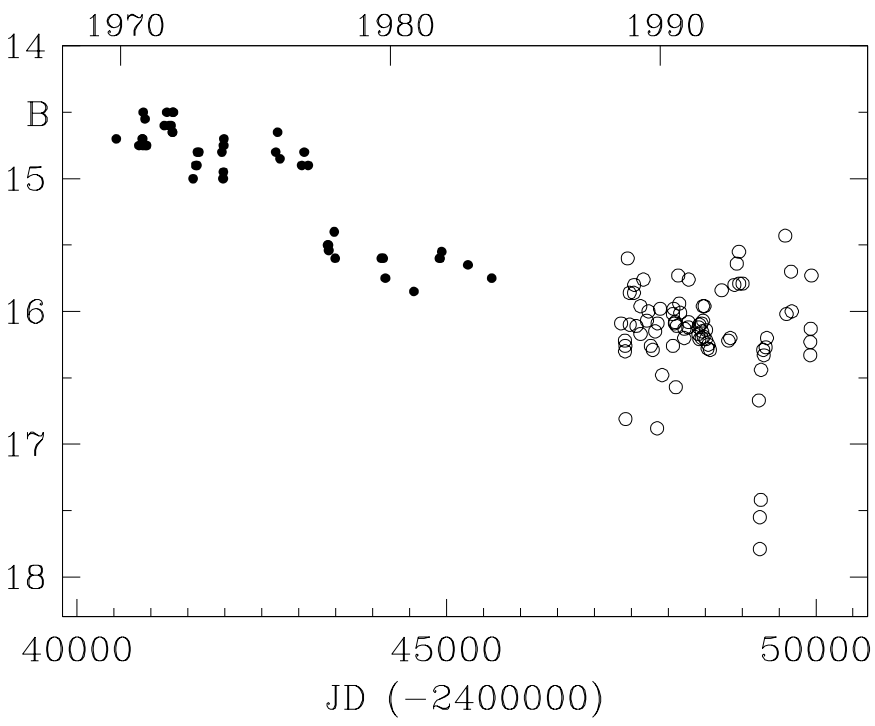

Fig. 2. $B$ lightcurve of V627 Cas (=AS 501). Dots: data from Table 1. Open circles: photoelectric photometry by Kolotilov et al. (1996).

cence level at $B \sim 15.3$, close to the 1999 value. A sudden brightening occurred at the very end of the observational period: its detection is solid given the careful check of the AS 323 image on the plate that excluded local defects.

The most interesting aspect of the AS 323 lightcurve is however the series of drops below the quiescent level. A search for periodicities has revealed several possible periods, the stronger one being $\mathrm{P}=197.6$ days. The $B$ data are phase-plotted against it in Figure 1, showing a lightcurve closely resembling a deep eclipsing binary $(\triangle B \sim 1.5$ mag). More data are however necessary to firmly establish the periodicity, refine the period and confirm the suspected eclipsing nature. Hopefully, similar programs could locate in other archives more plates containing AS 323 and solve the issue.

If the $\mathrm{P}=197.6$ days should be confirmed as the orbital period of AS 323, it would be the shortest known among symbiotic stars, with the closest cases being TX CVn (199 days), T CrB (228 days) and BD-21.3873 (282 days; cf. Belczyński et al. 2000). The M3 giant in AS 323 would then quite probably fill its Roche lobe and show the characteristic ellipsoidal distortion of its lightcurve.

\section{2. $V 627$ Cas}

According to Kolotilov et al. (1996), V627 Cas (=AS 501) is an unusual type of symbiotic star because it could harbor a post-AGB cool giant. During the post-AGB phase a star is supposed to evolve very rapidly. The secular decrease in brightness evident in Figure 2 could be then ascribed to global modifications of the cool giant that dominates the emission of V627 Cas in the $B$ band.

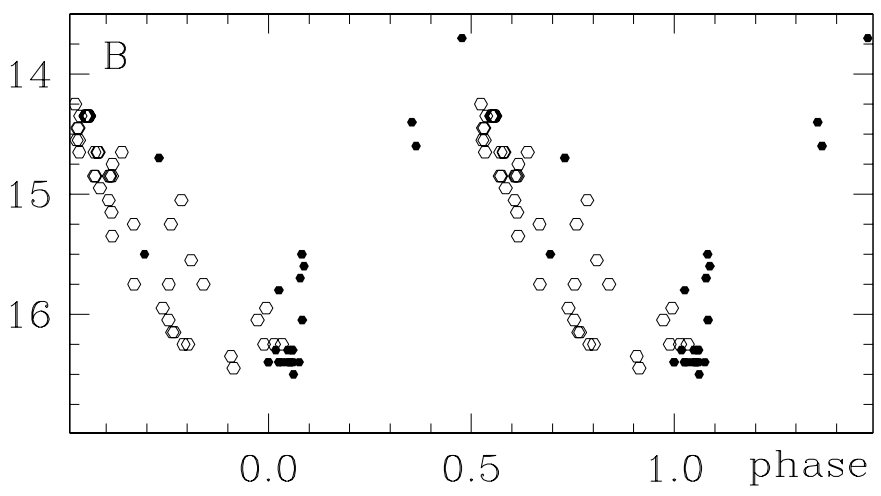

Fig. 3. The $B$ data of $\mathrm{CM}$ Aql folded onto a $\mathrm{P}=1058$ day period. Dots: data from Table 1. Open circles: data from Harwood (1925).

Kolotilov et al. (1996) also reported on a small amplitude pulsation of the cool giant with a period of $\mathrm{P}=466$ days. This periodicity (or any other else) does not seem to be present in the $B$ or $I$ band data of Table 1 , which cover an earlier time interval. The fact that the cool giant may have started to pulsate on such short time scales may again argue in favor of the Kolotilov et al. (1996) scenario of a post-AGB, rapidly evolving star.

\section{3. $C M A q l$}

According to the extensive literature search by Kenyon (1983), CM Aql has varied in the past between $16.4 \geq$ $B \geq 13.2$, with outbursts recorded in 1914, 1925 (when it was discovered), 1934 and 1950.

Our data in Table 1 extend over $16.5 \geq B \geq 13.7$, the same as in older records, without evidence for separated quiescence and outburst phases and favoring instead a continuous variability. It has also to be noticed that at the time of the 1925 "outburst" HeII 4686 was in strong emission (Harwood 1925), contrary to the typical behavior of symbiotic stars.

We argue that the variability so far observed in CM Aql is not modulated by outbursts, but it is instead periodic in nature. In Figure 3 the data of Harwood (1925) and those of Table 1 are phase plotted according to a period of $\mathrm{P}=1058$ days. The sinusoidal shape would suggest a reflection effect interpretation. A $\triangle B \sim 2$ mag amplitude would however be unusually large for a symbiotic star. Clearly, further data from other plate archives are necessary to firmly address the period, the nature of the sinusoidal-like variability and the absence of outbursts.

\section{References}

Acker A., Lundström I., Stenholm B. 1988, A\&AS 73, 325

Belczynski K., Mikolajewska J., Munari U., Ivison R. J., Friedjung M. 2000, A\&AS 146, 407 
Caldwell J.A.R., Cousins A.W.J., Ahlers C.C., van Wamelen P., Maritz E.J. 1993, SAAO Circ. 15, 1

Harwood M. 1925, Bull. Harv. Coll. Obs. N. 826

Henden A., Munari U. 2000, A\&AS 143, 343

Henden A., Munari U. 2001, A\&A, submitted

Kenyon, S.J. 1983, Ph.D. thesis, Univ. of Illinois UrbanaChampaign

Kenyon, S.J. 1986, The Symbiotic Stars, Cambridge Univ. Press

Kolotilov E.A., Munari U., Yudin B.F. 1995, A\&A 293, 815

Kolotilov E.A., Munari U., Yudin B.F., Tatarnikov A.M. 1996, Astron.Rep. 40, 812

Mikolajewska J., Acker A., Stenholm B. 1997, A\&A 327, 191

Mikolajewski M., Mikolajewska J., Khudyakova T.N. 1990, A\&A 235, 219

Munari U. 1992, A\&A 257, 163

Munari U., Rejkuba M., Mattei J., Hazen M., Luthardt R., Yudin B.F. 1997, A\&A 323, 113

Sabbadin F. 1986, A\&AS 65, 301 